REVIEW

\title{
Risk reduction counselling for prevention of sexually transmitted infections: how it works and how to make it work
}

\section{A Rietmeijer}

Sex Transm Infect 2007;83:2-9. doi: 10.1136/sti.2005.017319

Prevention research in the past decade has proved the efficacy of risk reduction counselling in reducing the risks for sexually transmitted infections (STIs). The question currently facing STI service providers is therefore not so much whether counselling should be part of the standard of STI care but rather how this intervention can be implemented given the logistical and resource constraints of a busy practice setting. After a brief introduction of the history and an overview of the models for risk reduction counselling and their theoretical and scientific underpinnings, the focus of this paper will be on the extent to which individual prevention models have been adopted in different clinical settings, the impediments to implementation and suggestions for improvement.

Correspondence to: C A Rietmeijer, Denver Public Health Department, 605 Bannock Street, Denver, CO 80204-4507, USA; kees.rietmeijer@ dhha.org

Accepted 12 June 2006 Published Online First 28 June 2006
B efore the onset of the HIV epidemic, prevention of sexually transmitted infections (STIs), particularly gonorrhoea and syphilis, focused on case finding and treatment. Although prevention campaigns as part of the social health movement in the early part of the last century had warned the public of the dangers of venereal disease and its vectors, in which prostitution was prominently featured, it was the discovery of penicillin that promised to break the back of the epidemics of gonorrhoea and syphilis that the Second World War had spawned. The ushering in of the antibiotic era resulted in a focus on testing and treating rather than on reducing high-risk sexual behaviours as the primary means of preventing STI transmission. In fact, as a result of the sexual revolution in the 1960s, sexual behaviour had become riskier, largely as a result of earlier onset of sexual activity and increasing numbers of sex partners. To the extent that there was a "condom code" in this era, it was largely driven by contraceptive desires rather than prevention of STI. Increasing rates of STIS followed, particularly among men who have sex with men (MSM), for whom the sexual liberation not only included increased numbers of sex partners but also an expansion of their sexual repertoire, including importantly, increasing engagement in anal sex. ${ }^{1}$ Many MSM experienced multiple episodes of gonorrhoea and syphilis, but they were often seen as "risks of the trade" by both the patient and the doctor. During the late 1970s, the public was becoming increasingly aware that STIs were not limited to syphilis and gonorrhoea, but that there were other infections, such as chlamydia, as well as viral STI including hepatitis B, genital herpes and human papillomavirus infections, that could lead to serious sequelae. Still, even with genital herpes being touted as a serious and at the time untreatable condition that could lead to devastating effects on the newborns of infected mothers, and media attention given to this new "scarlet letter", 2 it was not until the advent of the incurable and ultimately deadly HIV infection that prevention of transmission through behavioural adjustment was taken seriously. People at risk for HIV infection, particularly MSM, soon realised the devastation of this new STI, and abandoned the idea of gonorrhoea and syphilis being "business as usual". Rapid adoption of safer sex behaviours, including reduction in number of partners and, reluctantly, condom use, resulted in a dramatic decrease in the incidence of gonorrhoea and syphilis in this population. These early changes were the direct result of population awareness and not of public health interventions aimed at reducing high-risk behaviours. $^{34}$

\section{HIV TESTING AND COUNSELLING}

The adage of testing and treating did not immediately apply for HIV infections. Although the causative virus of AIDS had been discovered in 1983 and the first commercial test was available in 1985, HIV testing was not widely adopted and, in fact, actively discouraged by some groups. These attitudes led to testing disparities between countries, which have persisted to date. Without the availability of effective treatment, a diagnosis of HIV was seen as a death sentence. Early research seemed to indicate that the knowledge of having HIV infection was associated with a reduced risk of transmission, but the burden of the diagnosis and the associated stigma prevented many high-risk people from taking the test. In the public health environment, there was also a movement away from the testing paradigm, which was replaced by a focus on behavioural risk reduction to avoid HIV transmission. The decade between 1985 and 1995 thus saw a tremendous effort in behavioural research that resulted in numerous prevention interventions at the individual, group and community levels. Some, but not all, involved HIV testing as an integral part of the intervention. Moreover, in this context, HIV testing was not just seen as an opportunity for people to learn their

Abbreviations: CDC, Centers for Disease Control and Prevention; MSM, men who have sex with men; RE-AIM, Reach, Efficacy, Adoption, Implementation and Maintenance; STI, sexually transmitted infection 
serostatus but rather as a teachable moment during which a person might be susceptible to interventions aimed at behaviour change. In 1986, the Centers for Disease Control and Prevention (CDC) published its first guidelines that highlighted the importance of offering voluntary testing and counselling, ${ }^{5}$ followed by an update in 1987 that emphasised the importance of decreasing testing barriers and disclosure of personal information. ${ }^{6}$ The CDC first published the counselling model that underlies most of the currently offered prevention counselling interventions in 1993. ${ }^{7}$ This model emphasised an interactive rather than a didactic interaction between counsellor and client (see box). Owing to the far-reaching implications of these recommendations, including costs involved and the absence of any proof that such counselling had the desired effect, studies were designed to prove the efficacy of counselling for HIV prevention.

\section{PROJECT RESPECT}

Arguably, the single most important study establishing the efficacy of prevention counselling, was Project RESPECT. ${ }^{8}$ Owing to its importance, particularly for STI prevention in those without HIV, we describe the details of its methods and results. Project RESPECT was a CDC-sponsored study conducted between 1994 and 1997 at STI clinics in five cities in the US: Baltimore, Denver, Newark, Long Beach and San Francisco. It was a randomised, controlled trial that enrolled 5758 heterosexual, HIV-negative patients attending STI clinics to evaluate the efficacy of three different counselling interventions conducted in conjunction with HIV testing. Routine HIV testing at that time involved two sessions: a pre-testing session during which prevention counselling was conducted and blood drawn for HIV testing using ELISA and a post-test session usually 710 days later, during which results and additional counselling was given. Interventions in Project RESPECT were tailored to this pre-test-post-test model. The control arm in this study consisted of standardised, short, personalised educational messages about HIV and STI at both the pre-test and posttest sessions, each about $5 \mathrm{~min}$ in duration. At the time of the study, this was considered to be the predominant mode of counselling given at the time and thus the "standard of care". The "brief prevention counselling" arm consisted of two 20min sessions that followed the prevention counselling model (see box). Finally, a third arm, "enhanced counselling", comprised a 20-min pre-test counselling session similar to the "brief prevention counselling" arm, followed by three l-h individual counselling sessions during the ensuing weeks that were based on the theories of reasoned action and social cognitive theory, ${ }^{9}$ and focused on attitudes, self-efficacy and social norms regarding condom use as well as other safe sex behaviours, including reduction in number of sex partners and discussing HIV/STI testing with sex partners.

All interventions were highly scripted, and multiple training and quality-assurance sessions were conducted to ensure that the interventions were delivered as intended and that intercounsellor variations were kept at a minimum. ${ }^{10}$ A wide array of sexual risk and preventive behaviours were evaluated at baseline, and at 3, 6 and 12 months after enrolment. Biomedical outcomes that included testing for the most common bacterial and viral STIs were evaluated at visits at 6 and 12 months. The results were encouraging. Compared with the control arm, the brief prevention counselling resulted in a $30 \%$ reduction in incident STI at 6 months and a $20 \%$ reduction at 12 months. Interestingly, the four-session enhanced counselling only marginally increased the STI reduction effects of the two prevention counselling sessions, and these differences were not statistically significant. ${ }^{8}$

\section{THEORETICAL CONSIDERATIONS AND OTHER PREVENTION COUNSELLING MODELS}

During the past 20 years, research to reduce risks for HIV and other STIs has been guided by several theories on behaviour change, particularly the health belief model, ${ }^{11-13}$ the theory of reasoned action, ${ }^{14}$ the theory of planned behaviour ${ }^{15}$ and the social cognitive theory. ${ }^{16-18}$ A detailed discussion of these theories is outside the scope of this paper and the reader is referred to the literature discussing these theories in the context of STIs and HIV. ${ }^{19-21}$ In general terms, these theories have proposed an array of psychological factors that have a critical bearing on behaviour and behaviour change. These factors include the following:

- From the health belief model: perception of risk and vulnerability, and the balance between anticipated positive and negative outcomes of performing a preventive behaviour

- From the social cognitive theory: modelling of behaviours by peers and important others, and self-efficacy (the sense that we can control our behaviour under a variety of circumstances)

- From the theories of reasoned action and planned behaviour: attitudes and perceived social norms influencing the intention to perform a certain behaviour, and perceived behavioural control. ${ }^{19}$

In addition, several models of behaviour change that partly incorporate concepts from the above-cited theories have been used in the context of STI/HIV prevention counselling, and will therefore be discussed in some detail.

Although prevention counselling as evaluated in Project RESPECT was not based on a single theory of behaviour change, certain elements from established theoretical models can be readily identified. Foremost is the recognition that risk reduction is a stepwise rather than an all-or-nothing process. This concept is reflected in the development of a tailored prevention plan by the client, assisted by the counsellor (see box). The stepwise progression of behaviour change has best been explored in the transtheoretical model of behaviour change, better known as the "stages-of-change" model. ${ }^{22}$ Briefly, this model describes behaviour change as a dynamic process of five successive stages: (1) pre-contemplation, where no change is contemplated; (2) contemplation, where longrange intentions to change are being formulated; (3) preparation, with short-term intentions to change; (4) action, where change has been implemented for a short duration; and (5) maintenance, where durable behaviour change has occurred. In addition to this dynamic, the model further suggests that different processes in behaviour change, such as raising consciousness and self-reinforcement, are necessary at different stages. Thus, interventions focusing on cognitive and emotional factors will be most influential in early stages, whereas actionoriented approaches are more effective in later stages. ${ }^{19}$ The concepts from the transtheoretical model are used somewhat implicitly in the brief prevention counselling model in Project RESPECT. A more formal stage-based HIV/STD prevention counselling model, that otherwise incorporates many of the same prevention counselling principles, has also been developed. In this model, the risk elicitation phase is used to "stage" a client for a certain behaviour (eg, condom use for vaginal sex with a casual partner), after which the counsellor uses the corresponding processes of change in an effort to move the client to the next phase. ${ }^{23}$

Another stage-based model that has many similarities with the transtheoretical model is the AIDS risk reduction model. ${ }^{24}$ This model uses constructs from the health belief model and social cognitive theory, and recognises the following beha- 


\section{Box 1: Principles of prevention counselling}

- Keep session focused on HIV/sexually transmitted infection (STI) risk reduction:

- Counselling should be tailored to deal with personal risk of the client rather than provision of predetermined counselling messages

- Counsellors should not be distracted by the client's additional, unrelated problems

- Use open-ended questions, role-play scenarios, attentive listening, and non-judgemental and supportive approaches to encourage the client to remain focused on personal risk reduction

- Conduct an in-depth, personalised risk assessment

- Assist the client in identifying concrete, acceptable measures of risk reduction:

- Explore previous risk reduction efforts and identify successes and challenges

- Acknowledge and support positive changes already made:

- Enhance the clients' beliefs that change is possible

- Clarify critical misconceptions:

- Focus on misconceptions verbalised by the client and avoid general discussions

- Negotiate a concrete, achievable step in behaviour change that will reduce HIV/STI risk:

- Risk reduction steps must be acceptable to the client

- In case of multiple risks, focus on the behaviour the client is most willing to change

- Risk reduction does not always involve a personal risk behaviour - for example, talking to a partner about his or her HIV serostatus or motivating the partner to be tested

- Identify barriers and facilitators in achieving the behavioural goal

- Referral to additional prevention and support services may be necessary

vioural stages: labelling (recognition of risk and vulnerability), commitment to behaviour change (through changes in attitudes and self-efficacy) and enactment and help seeking (including self-help and help from others). As in the transtheoretical model, the AIDS risk reduction model recognises processes by which people move from one stage to the next, specifically internal motivators (eg, negative emotions such as anxiety and fear) and external motivators, including social support. Interventions specific to the labelling phase are those aimed at increasing a person's perception of risk, those focusing on improving the perceived cost-benefit ratio are thought to be more important for the commitment phase, whereas at the enactment phase, interventions should focus on support mechanisms. ${ }^{20}$

A critical characteristic of the prevention counselling model is the importance of the counsellor-client interaction to negotiate the risk reduction plan. In this interaction, the counsellor is much more than a passive listener in the Rogerian sense.
Box 1 continued: Principles of prevention counselling

- Provide skill-building opportunities:

- Role play

- Condom demonstration

- Use explicit language in providing test results:

- Avoid in-depth technical discussions that may diffuse the prevention message

- Develop and implement a written counselling protocol:

- Keeps clinicians or counsellors and supervisors on task

- Can include examples of open-ended questions and risk reduction steps

- Should be part of clinic standing orders

- Ensure support by supervisors and administrators:

- Provide ongoing training opportunities

- Include in-performance evaluations

- Avoid using counselling sessions for data collection:

- If possible, complete paperwork at the end of the counselling session

- Checklist risk assessments are detrimental to effective counselling-relevance of routinely collected data should be periodically assessed

- Avoid the provision of unnecessary information:

- Discussion of theoretical risks may shift the focus away from the clients' risk situation and may cause them to lose interest

Adapted from the Centers for Disease Control and Prevention's Revised guidelines for HIV counseling, testing, and referral. ${ }^{30}$

Rather, the counsellor actively engages the clients in focusing on an achievable step towards behaviour change and makes pro-active suggestions to assist the clients in establishing their plan. In this regard, the client-centred model resembles the concepts forwarded in the information, motivation and behavioural skills model. According to this model, people are more likely to adopt STI/HIV preventive behaviours to the extent that they are well informed, motivated to act and possess the required skills to act effectively. ${ }^{20}$ In counselling interventions based on this model, the technique of motivational interviewing $^{25}$ is used to assess the importance that people ascribe to changing a certain behaviour (often rated on a scale from 1 to 10 ), and the confidence they have that such change is possible (also rated on a scale from 1 to 10). The counsellor then, with the client, explores what it would take to increase these ratings and thereby determines the deficits in information, motivation and behavioural skills that can be dealt with and modified in the intervention. ${ }^{26}$

Clearly, from the above discussion, although there are many differences in the details of each of these models, there are also important overlaps. As we move from the theoretical and research arena into the real world of intervention for STI/HIV prevention, it will be increasingly important to emphasise the 
commonalities between these models. This the most pragmatic approach, as the use of these common theoretical elements and methods are probably responsible for the efficacy of an individual intervention model and thus enhance the likelihood of positive outcomes in real-world interventions. These commonalities can be summarised as follows. Firstly, all models emphasise the critical importance of engaging the client into the intervention and are in that sense client centred. This is accomplished by individualising risk assessment, targeting behaviours the client is willing to change and developing a risk reduction plan with the client that he or she considers feasible and achievable. The second commonality, more or less explicitly stated in each of these models, is that change is a dynamic process in which stages may be recognised and in which small steps will ultimately lead to the desired outcome. Thirdly, it is recognised that the progression through these stages is influenced by factors that must be specific for each stage and need to be tailored to the client's capabilities and circumstances. Finally, these theories and models emphasise psychological processes and do not pay much attention to situational, social and societal factors that influence the behaviour change process and may be of particular importance in counselling for STI prevention.

\section{THE CHALLENGE OF TURNING RESEARCH INTO PRACTICE}

The overall conclusion from the earlier discussion is that effective models for behavioural counselling exist and that they may have a major effect on the incidence and recurrence of STI. However, given that most of the research was conducted almost a decade ago, it is fair to ask how these promising research findings have affected the daily practice of STI/HIV prevention. Although no data are available on the adoption of and adherence to the CDC prevention guidelines in the US, it seems that widespread implementation, particularly in the STI clinic setting, has not been accomplished. In the next section, the hurdles to implementation and maintenance of prevention counselling will be discussed for three prevention settings: HIV counselling and testing sites, STI clinics and HIV care settings. Implications for prevention counselling in the primary care setting will also be discussed. In addition to the state of prevention counselling, this discussion provides insights into the continuously evolving philosophy of STI/HIV prevention. I do not intend to merely paint a bleak picture of the overall state of affairs of prevention counselling. Rather, several settingspecific suggestions will be made that may help in overcoming the impediments to a more widespread adoption of an important intervention.

Importantly, in this context, problems with the translation and dissemination of effective interventions from research into daily practice are not limited to counselling for HIV/STI prevention or, for that matter, other interventions aimed at changing high-risk sexual behaviour. In fact, a body of research on this topic is emerging from the field of health promotion and prevention of chronic diseases that may provide a useful guide when considering the transfer, translation and dissemination processes. A central theme in this discourse is the dichotomy between efficacy, defined as whether a programme does more good than harm when delivered under optimum (research) conditions, and effectiveness, defined as whether a programme does more good than harm under real-world conditions. ${ }^{27}$ Glasgow et $a l^{28}$ proposed that trials on intervention efficacy and real-world effectiveness differ fundamentally along five dimensions using the RE-AIM evaluation framework. In this framework, R(each) refers to the representativeness of participants who are likely to be homogeneous and highly motivated in efficacy trials but much more heterogeneous in effectiveness settings. E(fficacy or effectiveness) relates to the type and effect of an intervention that is likely to be more standardised, intensive and delivered in ways to maximise effect size in efficacy trials, while tending to be brief, feasible, adaptable and not requiring great expertise in effectiveness settings. A(doption) concerns the setting or agency implementing the intervention. In efficacy research, involved settings have many resources and high-quality staff who are usually limited in number to reduce variability. At the effectiveness level, interventions must appeal to multiple and varied settings, and be able to be adapted to fit the setting's limitations. $\mathrm{I}($ mplementation) refers to the quality and consistency with which the intervention is delivered, both of which are usually high and well-controlled by research staff in efficacy trials but, much more variable where a variety of staff have competing demands settings in effectiveness. Finally, M(aintenance) and costs are usually not an issue at the efficacy level; resources are abundant and sustenance beyond the trial period is not of concern. By contrast, costs are a major consideration for programme effectiveness; many interventions are too costly as designed to be implemented and maintained in real-world (public health) settings.

Glasgow et al $^{28}$ also point to the deficiencies of the traditional, linear model, which begins with efficacy research and, through a "trickle-down" process, leads to changes in public health practice. Rather, they advocate for a greater focus on the end users-that is, the (public health) service providers-and involve them in a participatory research process to focus on effectiveness rather than efficacy. ${ }^{28}$

In keeping with the RE-AIM principles and recognising the importance of adhering to the core elements of an intervention (fidelity) while allowing adaptations to particular settings and populations, the CDC is currently supporting widespread dissemination of behavioural interventions for STI/HIV prevention through its Diffusion of Effective Behavioural

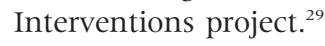

\section{SITES FOR HIV COUNSELLING AND TESTING}

HIV counselling and testing has long been the cornerstone of HIV prevention activities in the US, and public funding has been made available through the CDC to state health departments, and from there to local testing sites for many years. Counselling as part of the testing process has been a contingency of the funding process and, as has been described earlier in this paper, guidelines for counselling have been forwarded from the CDC since 1986. ${ }^{5}$ Counselling for prevention as evaluated in Project RESPECT was first described in $1993,{ }^{7}$ and updated most recently in 2001. ${ }^{30}$ Training for counselling for HIV prevention following these guidelines has been developed by the CDC and is conducted at the state level. Local testing sites that receive public funding are required to have their counsellors take these training courses as well as regular updates to maintain quality counselling skills. Still, testing and counselling occurs in a variety of settings, with a variety of resources and staff, and a variety of competing needs. As suggested by the RE-AIM framework, quality control in many settings is difficult and, although it is hoped that most counsellors will adhere to the very core of the prevention counselling principles, the level of control over the quality of the counselling process as assured in Project RESPECT and other efficacy trials will not be reached easily. Recent developments have put further emphasis on the quality of the counselling process. With the stagnation of HIV incidence in the US at 40000 new infections annually and the resurgence of high-risk behaviours among MSM, which at least in part seems to be related to changes in the perception of HIV and AIDS as a deadly condition in the era of highly active antiretroviral 
treatment, prevailing paradigms of HIV prevention have been revisited. As a result, a major shift has occurred from a focus on prevention of acquisition to an emphasis of prevention of transmission of HIV, and thus from prevention programmes that were mostly targeting those people currently not infected but at high risk, to programmes that target those who are already infected and are at risk for ongoing transmission. ${ }^{31}$ Therefore, an increasing focus has been placed on the identification of people with HIV infection and linking them to a comprehensive array of care and prevention services. Interestingly, and to some distressingly, as HIV infection is becoming a more treatable condition, the public health response seems to be a reversion to the traditional "test and treat" paradigm and a steering away from primary prevention through behaviour change that had become the dominant prevention response in the early phase of the HIV/AIDS epidemic.

In the context of linking people with HIV infection to care and prevention, one of the major problems with the traditional, two-step testing and counselling process is that a large proportion (up to $30 \%$ in certain studies) of people with positive test results do not return for post-test counselling, and thus remain unaware of their infection status and continue to be at risk for transmission. ${ }^{31}$ The introduction and promulgation of rapid HIV testing, where (preliminary) test results are available in $30 \mathrm{~min}$ after collecting a blood or saliva specimen, virtually guarantees provision of (preliminary) test results to the client, and thus provides a considerable improvement in the testing process. Importantly, rapid testing has been well received by both providers and clients and, as a result, many testing sites have switched to the rapid testing process. ${ }^{32}$ However, when the introduction of rapid testing in the US was contemplated, it was unclear as to whether counselling for prevention would be as effective in the context of rapid testing compared with the traditional two-step process. In anticipation of the availability and implementation of rapid testing, the CDC conducted a study to compare the intervention effects of both types of testing in a study design similar to Project RESPECT, and implemented by three of the five original Project RESPECT study sites. The results of Project RESPECT-2 showed that in the intention-to-treat analysis, intervention effects as measured by incident STIs subsequent to the intervention were similar between the two arms. Interestingly, however, in subanalyses, traditional two-step testing seemed to be more effective among men, and particularly among MSM. ${ }^{33}$ However, the results of Project RESPECT-2 did not receive the same amount of attention as Project RESPECT-1. In the context of identifying more people with HIV infection at an earlier stage of their infection, the quantity and quality of counselling for HIV prevention is probably being less emphasised. Indeed, in its Advancing HIV Prevention initiative, the CDC unequivocally states that "prevention counseling, although recommended for all persons at risk for HIV, should not be a barrier to testing" and "CDC will promote adoption of simplified HIV-testing procedures in medical settings that do not require prevention counseling before testing". ${ }^{31}$

Although the shift in focus from prevention counselling to HIV testing can be supported by numerous arguments, and although the test-and-treat paradigm offers the comfort of a traditional public health approach that is easier measured than behaviour change, it should be remembered that as an HIV prevention strategy, there is no scientific evidence to support its efficacy. Indeed, the focus on identification and linkage to care will probably reinforce the perception of HIV infection as a treatable condition, the very perception that many have linked to the recent resurgence of high-risk sexual behaviours among MSM. $^{44}$ Further, this approach is also predicated on the assumption that once in care, people with HIV infection will receive prevention services that will reduce the likelihood of ongoing transmission. ${ }^{35}$ As will be discussed later, this requires the participation of HIV care providers who have traditionally not been involved with risk reduction activities among their patients and for whom the added role of counsellor may or may not be a good fit.

Clearly, there is consensus that early identification of a person with HIV infection is beneficial from the perspective of both the individual's and the public's health. However, it would be a tragic mistake to solely rely on this strategy as the primary means of curbing the HIV epidemic.

\section{STI SETTINGS}

If the continued provision of quality counselling for prevention in HIV counselling and testing sites seems to be challenging, implementation in the STI clinical setting presents even greater obstacles. Although the CDC has supported prevention counselling in its STI treatment guidelines, ${ }^{36}$ to date, no sustained efforts have been made to develop comprehensive training and quality assurance plans for the incorporation of prevention counselling as a standard of care in US STI clinics. Obviously, such plans would require several resources that are currently not available. In addition, the shift away from prevention counselling in favour of increasing the number of people tested, as discussed earlier, has reduced the sense of urgency to change the status quo. As a result, in many clinics, even in the clinics that participated in Project RESPECT, sustained prevention counselling by clinicians has proved to be a great challenge indeed. Still, there is ample reason to reconsider improving the level of prevention counselling in STI clinics, either by clinicians or by other means. The single most important reason in favour of this is that available prevention research, and Project RESPECT foremost among them, has used incident STIs other than HIV as a proxy for and proof of the efficacy of HIV prevention interventions. This somehow has obscured the fact that prevention counselling has an important benefit for STI prevention in its own right. Indeed, participants in Project RESPECT were selected on the basis of their risk for STIs other than HIV, and the group at highest risk for HIV infection, MSM, was excluded from participation. Further, brief prevention counselling in this study was particularly effective among younger participants and among participants who had an STI at their enrolment visit into the study-that is, those at highest risk for subsequent gonorrhoea and chlamydia (re)infections. In a subanalysis of Project RESPECT, brief prevention counselling was associated with a $47 \%$ reduction of subsequent STIs among men and women aged $\leqslant 20$ years compared with those receiving standard educational messages, which translated into 9.1 STIs prevented per 100 people counselled. Similarly, among those with an STI at baseline, brief prevention counselling resulted in 5.3 STIs prevented per 100 people counselled. ${ }^{37}$ Unfortunately, resource limitations and other (perceived) barriers have discouraged serious consideration of how to implement this remarkably effective intervention. These barriers are important. Besides lack of buy-in by clinic supervisors and clinicians and the resources required to effectively train and maintain quality counselling among clinic staff, the single most important impediment to implementation seems to relate to clinic logistics. Although a "brief" prevention counselling requires "only" two 20-min pre-test and post-test counselling sessions, which, according to Project RESPECT-2 findings may be safely reduced to a single session, such additional time commitment over and above a typical 30-45-min visit is relevant and may be prohibitive given the existing patient workload and staff constraints. However, rather than rejecting prevention counselling outright as impractical for the STI clinic 
setting, ways may be explored through which the lessons of research on the efficacy of prevention counselling may be incorporated in the standard of care and thus enhance the effectiveness of STI prevention.

Firstly, prevention counselling should not be seen as a timeintensive add-on to the clinic encounter but rather as a new way of interacting with a client. For example, risk assessments are routinely conducted in STI clinics and are usually driven by several scripted, closed-ended questions in the clinic chart. These questions may be useful to generate clinic statistics and long-term trends, but are much less so for the purposes of risk reduction. ${ }^{30} \mathrm{~A}$ more open-ended risk assessment can quickly provide insight into a particular patient's specific risk, the stepping stone towards a risk reduction plan, and generate statistical information at the same time. By the same token, clinicians most often do counsel their clients, and indeed may go great lengths in doing so. However, such counselling is often driven by the clinician's perception of the client's risk and is often in the form of (well-meaning) advice on how to reduce such risks. Rather, this time could be used to engage the client in a risk reduction plan based on behaviours identified by the client and his or her readiness for change. Thus, training of clinicians in the basic concepts of prevention counselling could replace the traditional, one-sided, clinician-patient encounter with a more client-centred interaction that should not necessarily take more time but could prove to be much more effective in achieving the desired outcome of risk reduction. Despite the evidence to the contrary, many STI providers still do not believe that prevention counselling can be effective, and are thus reluctant to change their practice behaviours. Changing provider behaviours may indeed be as challenging as changing the behaviours of their clients. Thus, efforts should be undertaken to educate providers about the efficacy of prevention counselling and the change they can help accomplish in the lives of their patients. In this context, the US National Network of STD/HIV Prevention Training Centers (http:// stdhivpreventiontraining.org) should be mentioned as an important training resource.

In addition to training clinicians about the basics of prevention counselling, other strategies and resources may be used to improve the level of prevention counselling in STI clinics. For example, offering prevention counselling could be limited to those clients at highest risk or to those for whom this intervention seems to be most effective, including adolescents or young adults, and patients with a previous STI. ${ }^{37}$ Further, counselling does not necessarily have to be provided by the clinician but could be deferred to specifically trained counsellors in the clinic. If clinics have onsite or nearby HIV testing and counselling sites, counsellors from these sites may be shared to offer counselling in the STI clinic, and, as argued above, not necessarily limited to those clients receiving HIV testing. To the extent that HIV counsellors are increasingly offering other STI testing (such as syphilis and gonorrhoea) as part of their prevention "tool kit", both in clinical and in outreach settings, such an integration between HIV and STI prevention services seems to be occurring with increasing frequency. Where HIV prevention counsellors are benefiting from the expertise of STI clinicians, the STI clinic may benefit greatly from the extensive experience of HIV prevention counsellors.

Increasingly, sophisticated computer and web-based technologies offer other possibilities. The concepts of tailoring prevention messages to individual risk behaviours and developing stepwise prevention plans, both core elements of prevention counselling, have been used to develop brief computerised interventions that can be conducted without the use of a counsellor. On the basis of a computer-based risk assessment, computer algorithms can be used to develop highly personalised risk reduction strategies. In one study, such technologies were applied to develop tailored prevention messages in the form of print materials that proved to be effective in reducing high-risk behaviours among adolescent females. ${ }^{38}$ Other studies have developed tailored interventions that are conducted entirely online. ${ }^{39} 40$ A CD Rom product based on the principles of prevention counselling for use in the clinical setting is in the final stages of development. ${ }^{41}$

\section{PREVENTION IN HIV CARE SETTINGS}

The shift in focus in HIV prevention from acquisition by previously uninfected people to transmission by those infected is also shifting a major burden of prevention on to the shoulders of the HIV care provider. In the past few years, numerous activities have been deployed to engage HIV care providers in ongoing prevention efforts with their HIV-infected clientele. Whereas the HIV care provider is an obvious ally in the battle against HIV, provision of prevention services is not something that necessarily comes naturally to medical providers in general, and to HIV care providers in particular. Firstly, engaging people living with HIV/AIDS in ongoing risk assessments and confronting them with the fact that they may put others at risk for infection may be seen by some providers as antagonistic in the patient-doctor relationship and counter to the patient advocacy role they prefer to have. Secondly, clinic logistics and resources may limit the amount of services that can be provided, and adding ongoing prevention services may be seen as unfeasible, especially if there is no source of reimbursement for the provision of these services. Finally, there is a paucity of research to suggest that individual prevention counselling in care settings may be successful. One trial has shown that the incorporation of brief prevention messages (particularly those that focus on the negative effects of ongoing risk behaviours) into the standard of HIV care may reduce risk behaviours among those with HIV infection. ${ }^{42}$ Another intervention, based on the information, motivation and behavioural skills model, has been described and shown to be acceptable to patients and providers. ${ }^{26}$ An efficacy trial to show the positive effect on self-reported risk behaviours was recently published. ${ }^{43}$ Still, the importance of shifting the prevention paradigm to the person with HIV infection has caused a level of impatience in awaiting more efficacy trials, and has prompted a movement to apply lessons from past experience directly into practice-based interventions. Taking a cue from the RE-AIM model, rather than developing controlled prevention trials, the focus seems to be on working with organisations that have provided training and capacity building to care providers to develop interventions that are built on the best that prevention research has to offer, yet is acceptable at the provider level. For example, in the US, the CDC-funded STD/HIV Prevention Training Centers have been funded to collaborate with the AIDS Education and Training Centers (funded by the Health Resources Services Administration) in developing a training curriculum for HIV care providers to implement brief ongoing counselling with their patients. The "Ask, Screen, Intervene" curriculum is based on the principles of prevention counselling outlined earlier in this paper, and attempts to establish three prevention goals among providers: (1) to assess ongoing risk behaviours through the use of open-ended questions; (2) to screen for STIs; and (3) to develop a tailored, stepwise risk reduction plan for those at ongoing risk for HIV transmission. ${ }^{44}$ Similar projects are the CDC-sponsored HIV Prevention into Medical Care Settings demonstration project ${ }^{45}$ and the Prevention with HIV Infected Persons in Primary Care Settings project funded by the Health Resources and Service Administration, which is part of the Special Projects of National Significance programme. ${ }^{46}$ All three seem to have a common approach that is focused on developing 
brief, acceptable interventions based on existing prevention models, with a focus on acceptability, in the busy HIV care environment. Although this approach may be driven by the urgency of establishing prevention capacity in the care setting, this development is encouraging when looked at from the efficacy-effectiveness dynamic. Findings from these projects, both at the level of acceptance, implementation and sustenance by providers, as well as at the level of (sustained) behaviour change by patients, are awaited with great interest.

\section{IMPLICATIONS FOR THE PRIMARY CARE SETTING}

The emphasis of this paper has been on the provision of risk reduction counselling in specialised sessions: HIV counselling and testing sites, STI clinics and HIV care settings. Still, it is appreciated that a large proportion of STIs are diagnosed and treated in primary care settings. This discussion may be of interest to the primary care provider, but logistical and other constraints mentioned for specialised settings are also present in the primary care setting, and are compounded by the fact that primary care providers serve a much more diverse population and deal with a much larger array of healthcare problems than those encountered by providers of STI/HIV prevention and care. Thus, it is not surprising that many primary care providers do not think they are responsible for STI preventive services among their patients, and have generally negative attitudes towards the effectiveness of STI counselling. ${ }^{47}$ Even if these negative attitudes could be overcome, the major limiting factor for the inclusion of adequate counselling for STI risk prevention in primary care settings is the negative attitude held by many about the inclusion of yet another important intervention that is added to the burden and increasingly tight schedules of clinics that, particularly in areas with high STI incidence, are chronically under-funded. However, without trivialising this problem, there may be ways to tackle this issue from a different perspective. STI/HIV risk behaviours should not be seen as a categorical problem but rather as part of the spectrum of health risk behaviours that primary care clinicians encounter on a daily basis that include smoking and substance misuse, lack of exercise and eating disorders. The advantage of the models for prevention counselling is that they are not necessarily behaviour specific. Indeed, the theories that underlie these models have in large part been developed for other behavioural problems, including smoking cessation, weight control and psychological distress, ${ }^{48}$ long before the onset of the AIDS epidemic made them relevant for HIV/STI prevention. Prevention counselling, as discussed in this paper, thus finds an interesting corollary in the concept of patient-centred care in the literature on primary care that operates along similar lines to work with patients to agree on their most salient health problems and to take an incremental approach to setting attainable (behavioural) goals. ${ }^{49}$ In that sense, the nature of the health problem is secondary to the patient-centred approach taken by the care provider. Thus, in a patient who is at high STI/HIV risk, prevention counselling to reduce high-risk sexual behaviour is no longer an alien, timeconsuming add-on for the overburdened primary care provider but rather a natural outflow of a generic patient-provider interaction model.

Competing interests: None declared.

\section{REFERENCES}

1 Rotello G. Sexual ecology. New York: Penguin, 1997.

2 Wallis C. Battling an elusive invader. Time 1982;120:68.

3 Judson F. Fear of AIDS and gonorrhea rates in homosexual men. Lancet 1983;2:159-60.

4 Rietmeijer CA. Resurgence of risk behaviors among men who have sex with men: the case for HAART realism. Sex Transm Dis 2005;32:176-7.
5 Centers for Disease Control and Prevention. Current trends: additional recommendations to reduce sexual and drug abuse-related transmission of human T-lymphotropic virus type III/lymphadenopathy-associated virus. Morb Mortal Wkly Rep 1986;35:152-5.

6 Centers for Disease Control and Prevention. Perspectives in disease prevention and health promotion: Public Health Service guidelines for counseling and antibody testing to prevent HIV infection and AIDS. Morb Mortal Wkly Rep 1987;36:509-15

7 Centers for Disease Control and Prevention. Technical guidance on HIV counseling. Morb Mortal Wkly Rep 1993:42:5-9

$8 \mathrm{Kamb}$ ML, Fishbein M, Douglas JM Jr, et al. Efficacy of risk-reduction counseling to prevent human immunodeficiency virus and sexually transmitted diseases: a randomized controlled trial. Project RESPECT Study Group. JAMA 1998;280:1161-7.

9 Fishbein M, Bandura A, Triandis $\mathrm{H}$, et al. Factors influencing behavior and behavior change: final report-theorist's workshop. Rockville, MD: National Institute of Mental Health, 1992.

10 Kamb ML, Dillon BA, Fishbein M, et al. Quality assurance of HIV prevention counseling in a multi-center randomized controlled trial. Project RESPECT Study Group. Public Health Rep 1996;111(Suppl 1):99-107.

11 Becker MH. The health belief model and personal health behavior. Health Educ Monogr, 1974;2:324-473.

12 Rosenstock IM. The health belief model and preventive health behavior. Health Educ Monogr 1974;2:354-85.

13 Rosenstock I, Strecher V, Becker M. The health belief model and HIV risk behavior change. In: DiClemente R, Peterson J, eds. Preventing AIDS. Theories and methods of behavioral interventions. New York: Plenum Press, 1994.

14 Fishbein M, Ajzen I. Belief, attitude, intention and behavior: an introduction to theory and research. Boston, MA: Addison-Wesley, 1975.

15 Ajzen I. From intentions to actions: a theory of planned behavior. In: Kuhl J, Beckmann J, eds. Action-control: from cognition to behavior. Heidelberg, Germany: Springer, 1985:11-39.

16 Bandura A. Social learning theory. Englewood Cliffs, NJ: Prentice-Hall, 1977.

17 Bandura A. Social foundations of thought and action: a social cognitive theory. Englewood Cliffs, NJ: Prentice-Hall, 1986.

18 Bandura A. Exercise of personal agency through the self-efficacy mechanism. In: Schwartzer R, ed. Self-efficacy: thought control of action. Washington, DC: Hemisphere, 1992.

19 Fishbein M, Guinan M. Behavioral science and public health: a necessary partnership for HIV prevention. Public Health Rep 1996;111(Suppl):5-10.

20 Fisher JD, Fisher WA. Theoretical approaches to individual-level change in HIV risk behavior. In: Peterson J, DiClemente R, eds. HIV prevention handbook. New York: Kluwer Academic, 2000.

21 Kalichman S. Preventing AIDS: a sourcebook for behavioral interventions. Mahwah, NJ: Lawrence Erlbaum, 1998.

22 Prochaska JO, Velicer WF. The transtheoretical model of behavior change. Am J Health Prom 1997; 12:38-48.

23 Coury-Doniger $\mathrm{P}$, Levenkron JC, Knox KL, et al. Use of stage of change (SOC) to develop an STD/HIV behavioral intervention: phase 1. A system to classify SOC for STD/HIV sexual risk behaviors-development and reliability in an STD clinic. AIDS Patient Care STDS 1999;13:493-502.

24 Catania JA, Kegeles SM, Coates TJ. Towards an understanding of risk behavior: an AIDS risk reduction model (ARRM). Health Educ Q 1990;17:53-72.

25 Miller W, Rollnick S. Motivational interviewing: preparing people to change addictive behavior. New York: Guilford Press, 1991.

26 Fisher JD, Cornman DH, Osborn CY, et al. Clinician-initiated HIV risk reduction intervention for HIV-positive persons: formative research, acceptability, and fidelity of the options project. J Acquir Immune Defic Syndr 2004;37:S78-87.

27 Flay BR. Efficacy and effectiveness trials (and other phases of research) in the development of health promotion programs. Prev Med 1986;15:451-74.

28 Glasgow RE, Lichtenstein E, Marcus AC. Why don't we see more translation of health promotion research to practice? Rethinking the efficacy-to-effectiveness transition. Am J Public Health 2003;93:1261-7.

29 Diffusion of Effective Behavioral Interventions (DEBI). http:// www.effectiveinterventions.org/ (accessed 7 Oct 2006).

30 Centers for Disease Control and Prevention. Revised guidelines for HIV counseling, testing, and referral. MMWR Recomm Rep 2001;50:1-53.

31 Centers for Disease Control and Prevention. Advancing HIV prevention: new strategies for a changing epidemic-United States, 2003. Morb Mortal Wkly Rep 2003;52:329-32.

32 Spielberg F, Branson BM, Goldbaum GM, et al. Overcoming barriers to HIV testing: preferences for new strategies among clients of a needle exchange, a sexually transmitted disease clinic, and sex venues for men who have sex with men. J Acquir Immune Defic Syndr 2003;32:318-27.

33 Metcalf CA, Douglas JM Jr, Malotte CK, et al. Relative efficacy of prevention counseling with rapid and standard HIV testing: a randomized, controlled trial (RESPECT-2). Sex Transm Dis 2005;32:130-8.

34 Wolitski RJ, Valdiserri RO, Denning PH, et al. Are we headed for a resurgence of the HIV epidemic among men who have sex with men? Am J Public Health $2001 ; 91: 883-8$.

35 Centers for Disease Control and Prevention. Incorporating HIV prevention into the medical care of persons living with HIV. Recommendations of CDC, the Health Resources and Services Administration, the National Institutes of Health, and the HIV Medicine Association of the Infectious Diseases Society of America. MMWR Recomm Rep 2003;52:1-24.

36 Centers for Disease Control and Prevention. Sexually Transmitted Diseases Guidelines, 2006. Morb Mortal Wkly Repart 2006;55:3 
37 Bolu OO, Lindsey C, Kamb ML, et al. Is HIV/sexually transmitted disease prevention counseling effective among vulnerable populations? A subset analysis of data collected for a randomized, controlled trial evaluating counseling efficacy (Project RESPECT). Sex Transm Dis 2004;31:469-74.

38 Scholes D, McBride CM, Grothaus L, et al. A tailored minimal self-help intervention to promote condom use in young women: results from a randomized trial. AIDS 2003;17:1547-56.

39 Bull SS, Lloyd L, Rietmeijer C, et al. Recruitment and retention of an online sample for an HIV prevention intervention targeting men who have sex with men: the Smart Sex Quest Project. AIDS Care 2004;16:931-43.

40 Davidovich U, de Wit J, Stroebe W. The effect of an internet intervention for promoting safe sex between steady male partners - results of a longitudinal randomized controlled trial online. Paper presented at XV International AIDS Conference, 2004, Bangkok, Thailand.

41 Resources Online. http://www.ronline.com/care/ (accessed 7 Oct 2005)

42 Richardson JL, Milam J, McCutchan A, et al. Effect of brief safer-sex counseling by medical providers to HIV-1 seropositive patients: a multi-clinic assessment. AIDS 2004;18:1179-86.
43 Fisher JD, Fisher WA, Cornman DH, et al. Clinician-delivered intervention during routine clinical care reduces unprotected sexual behavior among HIV-infected patients. J Acquir Immune Defic Syndr 2006;41:44-52.

44 Thrun M, Hall C. Incorporating HIV prevention into the medical care of persons living with HIV-training care providers to "Ask, Screen, Intervene". Paper presented at 2005 National HIV Prevention Conference, 2005, Atlanta.

45 Centers for Disease Control and Prevention. Demonstration projects for primary care providers: incorporating HIV prevention into medical care settings (PICS). http://www.cdc.gov/hiv/partners/AHP/PICS.htm (accessed 7 Oct 2005).

46 HRSA. Special initiatives: prevention with HIV infected persons in primary care settings. http://hab.hrsa.gov/special/pop_overview.htm (accessed 4 Aug 42005).

47 Ashton MR, Cook RL, Wiesenfeld HC, et al. Primary care physician attitudes regarding sexually transmitted diseases. Sex Transm Dis 2002;29:246-51.

48 Prochaska J, DiClemente C. Common processes of self-change in smoking, weight control, and psychological distress. In: Shiffman S, Aslby Willis T, eds. Coping and substance use. Orlando, FL: Academic Press, 1985.

49 Lowes R. Patient-centered care for better patient adherence. Fam Pract Manag 1998;5:46-47, 51-44, 57.

\section{BNF for Children 2006, second annual edition}

In a single resource:

- guidance on drug management of common childhood conditions

- hands-on information on prescribing, monitoring and administering medicines to children

- comprehensive guidance covering neonates to adolescents

For more information please go to bnfc.org 
conflict with UK guidelines. Their preferred regimen for the treatment of pelvic inflammatory disease is in particular surprising (doxycycline and metronidazole) since it conforms with neither UK nor US guidelines and three studies have now shown it to be inferior to alternative regimens.

Overall Fast facts: sexually transmitted infections is to be recommended with just a few caveats.

Jonathan Ross

\section{CORRECTIONS}

doi: 10.1136/sti.2006.021782.corr 1

There was an error in the August issue of the journal (Dodds JP, Johnson AM, Parry JV, et al.
A tale of three cities: persisting high HIV prevalence, risk behaviour and undiagnosed infection in community samples of men who have sex with men. Sex Transm Infect 2007;83:392-6.) The last sentence on page 2 should read as follows: "All were screened by GACELISA HIV 1 and 2, whose sensitivity and specificity had been determined as $99.5 \%$ (95\% CI $97.1 \%$ to $99.9 \%$ ) and $99.7 \%$ (95\% CI $98.9 \%$ to $99.9 \%)$, respectively. ${ }^{13} 14$

doi: 10.1136/sti.2006.023283.corr 1

Several errors occurred in an article published in the July issue of the journal (Evans AR, Wiggins RD, Mercer $\mathrm{CH}$, et al. Men who have sex with men in Great Britain: comparison of a self-selected internet sample with a national probability sample. Sex Transm Infect 2007;83:200-5.) A corrected version of the article is available on our website at http:// sti.bmj.com/supplemental.

doi: 10.1136/sti.2006.023531.corr 1

Two articles from the August 2007 supplement were unlocked online but not in print. The articles are: Orroth KK, Freeman EE, Bakker R, et al. Understanding the differences between contrasting HIV epidemics in east and west Africa: results from a simulation model of the Four Cities Study. Sex Transm Infect 2007;83(Suppl 1):i5-i6 and Freeman EE, Orroth KK, White RG, et al. Proportion of new HIV infections attributable to herpes simplex 2 increases over time: simulations of the changing role of sexually transmitted infections in sub-Saharan African HIV epidemics. Sex Transm Infect 2007;83(Suppl 1):il7-i124. Both articles are freely available. 